\title{
Role of core needle biopsy for patients with indeterminate, fine-needle aspiration cytology
}

\author{
Woo Jung Choi $\cdot$ Jung Hwan Baek
}

Received: 13 September 2013/Accepted: 19 September 2013/Published online: 28 November 2013

(c) Springer Science+Business Media New York 2013

In this journal of Endocrine, Nasrollah et al. [1] reported a new core needle biopsy (CNB) technique for thyroid nodules classified as indeterminate in fine-needle aspiration (FNA) cytology. This new technique obtains core samples consisting of nodular tissue, extranodular parenchyma, and the nodule's capsule when it is present. They concluded that this technique is helpful to discern the nature of thyroid nodules classified as indeterminate on FNA.

In 1930s, large-needle biopsy using 14-gauge needle without ultrasound (US) had been used for diagnosis of thyroid nodules [2], but it showed no benefit in terms of its safety and efficacy as compared with those of FNA [3]. Therefore, in 1980s, FNA has been used as the standard technique for diagnosing thyroid cancer [4]. CNB has recently been reconsidered for use in the diagnosis of thyroid cancer because modern type, spring-activated CNB under US guidance improved both the safety and efficacy of this technique [5]. The patient comfort during and after CNB or tolerability of this procedure is well reported [6].

The majority of the recent guidelines have recommended CNB for patients with thyroid nodules with previously non-diagnostic FNA results [7-9] and in patients with suspicious lymphoma or anaplastic cancer [8, 9]. However, the American Thyroid Association guidelines recommend repeat FNA for patients with previously nondiagnostic FNA results instead of CNB [10]. Recently, Yeon et al. [11] retrospectively studied a large group of patients with non-diagnostic FNA results and concluded that $\mathrm{CNB}$ could reduce both the non-diagnostic and

W. J. Choi · J. H. Baek ( $₫)$

Department of Radiology and Research Institute of Radiology, Asan Medical Center, University of Ulsan College of Medicine, 86 Asanbyeongwon-Gil, Songpa-Gu, Seoul 138-736, Korea e-mail: radbaek@naver.com inconclusive results. Their results also showed that CNB could prevent unnecessary diagnostic surgery in $96 \%$ of the patients with repeated, non-diagnostic FNA results.

For indeterminate FNA results, F18-FDG-PET/CT or (99 m)Tc-MIBI scan may be helpful in patients with thyroid nodules in which malignancy is suspected on the basis of conventional diagnostic techniques [3,4]. There are no guidelines recommending CNB. Therefore, repeated, indeterminate results of FNA cause patients to undergo diagnostic surgery [8]. As thyroid FNA has become widely used, unnecessary surgery has increased. In a recent study by Park et al. [12], they retrospectively reported the role of CNB for indeterminate thyroid nodules. Their results showed that CNB is superior to repeat FNA for previously non-diagnostic or indeterminate nodules. In another report on this topic by $\mathrm{Na}$ et al. [13], they again prospectively evaluated and suggested that CNB can effectively reduce inconclusive results compared with repeat FNA in nodules with previously non-diagnostic or atypia of undetermined significance (AUS)/follicular lesion of undetermined significance (FLUS) FNA results. This may be related to the larger tissue sample and the additional histological information obtained by $\mathrm{CNB}$ as compared with FNA. Choi et al. [14] verified the value of CNB in a large study population $(N=191)$ of patients with AUS and FLUS seen in previous FNA results.

In a recent study by Nasrollah et al. [1], 20 patients were diagnosed as follicular neoplasm without false negative results by new $\mathrm{CNB}$ technique including nodular tissue, extranodular parenchyma, and the nodule's capsule. On histology, all but one patient was diagnosed as having follicular adenoma, while the other patient was diagnosed with follicular carcinoma. Adenomatous nodules lack or have a thin fibrous capsule, while follicular neoplasms usually manifest as thick, encapsulated lesions [15]. In 
these cases, CNB may be useful for the diagnosis as it obtains the sample including the rim of the nodule as well as extranodular tissue. These preliminary data demonstrate the important role of CNB for differentiating adenomatous nodules from follicular neoplasm.

Given these considerations, CNB may help to determine the nature of indeterminate, follicular-patterned thyroid nodules. Larger, prospective studies will be required for a better understanding of the efficacy of CNB using thingauge needles as well as the importance of cutting within the nodule border.

\section{References}

1. N. Nasrollah, P. Trimboli, L. Guidobaldi, D.D. Cicciarella Modica, C. Ventura, G. Ramacciato, S. Taccogna, F. Romanelli, S. Valabrega, A. Crescenzi, Thin core biopsy should help to discriminate thyroid nodules cytologically classified as indeterminate. A new sampling technique. Endocrine 43(3), 659-665 (2013)

2. H.E. Martin, E.B. Ellis, Biopsy by needle puncture and aspiration. Ann. Surg. 92(2), 169-181 (1930)

3. G. Treglia, C. Caldarella, E. Saggiorato, L. Ceriani, F. Orlandi, M. Salvatori, L. Giovanella, Diagnostic performance of $(99 \mathrm{~m}) \mathrm{Tc}-$ MIBI scan in predicting the malignancy of thyroid nodules: a metaanalysis. Endocrine 44(1), 70-78 (2013)

4. F. Bertagna, G. Treglia, A. Piccardo, E. Giovannini, G. Bosio, G. Biasiotto, K. Bahij el, R. Maroldi, R. Giubbini, F18-FDG-PET/ CT thyroid incidentalomas: a wide retrospective analysis in three Italian centres on the significance of focal uptake and SUV value. Endocrine. 43(3), 678-685 (2013)

5. S. Taki, K. Kakuda, K. Kakuma, Y. Annen, S. Katada, R. Yamashita, M. Kosugi, T. Michigishi, N. Tonami, Thyroid nodules: evaluation with US-guided core biopsy with an automated biopsy gun. Radiology 202(3), 874-877 (1997)

6. N. Nasrollah, P. Trimboli, F. Rossi, S. Amendola, L. Guidobaldi, C. Ventura, R. Maglio, G. Nigri, F. Romanelli, S. Valabrega, A. Crescenzi, Patient's comfort with and tolerability of thyroid core needle biopsy. Endocrine (2013). doi:10.1007/s12020-013-9979-x
7. Z.W. Baloch, E.S. Cibas, D.P. Clark, L.J. Layfield, B.M. Ljung, M.B. Pitman, A. Abati, The National Cancer Institute Thyroid fine needle aspiration state of the science conference: a summation. Cytojournal 5, 6 (2008)

8. H. Gharib, E. Papini, R. Paschke, D.S. Duick, R. Valcavi, L. Hegedus, P. Vitti, American Association of Clinical Endocrinologists, Associazione Medici Endocrinologi, and EuropeanThyroid Association Medical Guidelines for Clinical Practice for the Diagnosis and Management of Thyroid Nodules. Endocr. Pract. 16(Suppl 1), 1-43 (2010)

9. British Thyroid Association, Royal College of Physicians, Guidelines for the management of thyroid cancer, 2nd edn. (Royal College of Physicians, London, 2007)

10. D.S. Cooper, G.M. Doherty, B.R. Haugen, R.T. Kloos, S.L. Lee, S.J. Mandel, E.L. Mazzaferri, B. McIver, F. Pacini, M. Schlumberger, S.I. Sherman, D.L. Steward, R.M. Tuttle, Ata, Revised American Thyroid Association Management Guidelines for Patients with Thyroid Nodules and Differentiated Thyroid Cancer. Thyroid 19(11), 1167-1214 (2009)

11. J.S. Yeon, J.H. Baek, H.K. Lim, E.J. Ha, J.K. Kim, D.E. Song, T.Y. Kim, J.H. Lee, Thyroid nodules with initially nondiagnostic cytologic results: the role of core-needle biopsy. Radiology 268(1), 274-280 (2013)

12. K.T. Park, S.H. Ahn, J.H. Mo, Y.J. Park, D.J. Park, S.I. Choi, S.Y. Park, Role of core needle biopsy and ultrasonographic finding in management of indeterminate thyroid nodules. Head Neck J. Sci. Spec. 33(2), 160-165 (2011)

13. D.G. Na, J.H. Kim, J.Y. Sung, J.H. Baek, K.C. Jung, H. Lee, H. Yoo, Core-needle biopsy is more useful than repeat fine-needle aspiration in thyroid nodules read as nondiagnostic or atypia of undetermined significance by the Bethesda vystem for reporting thyroid cytopathology. Thyroid 22(5), 468-475 (2012)

14. Y.J. Choi, J.H. Baek, E.J. Ha, H.K. Lim, J.H. Lee, J.K. Kim, D.E. Song, Y. Shong, S.J. Hong, Different risk of malignancy and management recommendations in subcategories of thyroid nodules with atypia of undetermined significance (AUS) or follicular lesion of undetermined significance (FLUS): The role of US-guided coreneedle biopsy (CNB). Thyroid (2013). doi:10.1089/thy.2012.0635

15. S.R. Kini, Thyroid cytopathology: an atlas and text (Lippincott Williams \& Wilkins, Philadelphia, 2008) 